\title{
Editorial
}

\section{Time Delayed Equations as Models in Nature and Society}

\author{
Luca Guerrini, ${ }^{1}$ Luca Gori, ${ }^{2}$ Akio Matsumoto, ${ }^{3}$ Mauro Sodini, ${ }^{4}$ \\ Zizhen Zhang, ${ }^{5}$ and Carlo Bianca ${ }^{6}$ \\ ${ }^{1}$ Department of Management, Polytechnic University of Marche, 60121 Ancona, Italy \\ ${ }^{2}$ Department of Political Science, University of Genoa, 16125 Genoa, Italy \\ ${ }^{3}$ Department of Economics, Chuo University, 742-1 Higashi-Nakano, Hachioji, Tokyo, Japan \\ ${ }^{4}$ Department of Economics and Management, University of Pisa, 56124 Pisa, Italy \\ ${ }^{5}$ School of Management Science and Engineering, Anhui University of Finance and Economics, \\ Bengbu 233030, China \\ ${ }^{6}$ Laboratoire de Physique Statistique, Ecole Normale Supérieure, 75231 Paris, France
}

Correspondence should be addressed to Luca Guerrini; luca.guerrini@univpm.it

Received 4 April 2016; Accepted 4 April 2016

Copyright (c) 2016 Luca Guerrini et al. This is an open access article distributed under the Creative Commons Attribution License, which permits unrestricted use, distribution, and reproduction in any medium, provided the original work is properly cited.

Several phenomena analysed in both natural and social sciences models require the use of systems including current variables and lagged variables. The interest in retarded ordinary or partial differential equations has gained much attention recently because it has been seen that the introduction of time delays allows the capturing of more complex dynamics, thus enriching the description of the whole system. This special issue collects several manuscripts from people who work in different research fields. In total there were seventy-three submissions and only twenty-three were accepted for publication. The topics of the accepted papers range from theoretical studies to applications. In particular, there are new theoretical results on stochastics and fuzzy time delayed dynamic systems, on global stability, and on delay partial differential equations. With regard to the articles that focused on specific models, we mention here some interesting applications on disease dynamics, economic growth and business cycles, population dynamics, ecological systems, neural networks, and physics problems.

\section{Acknowledgments}

The guest editors would like to express their gratitude to all the authors and reviewers who took part in the success of this special issue.
Luca Guerrini

Luca Gori

Akio Matsumoto

Mauro Sodini

Zizhen Zhang

Carlo Bianca 


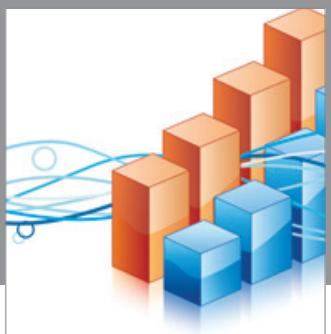

Advances in

Operations Research

vatem alat4

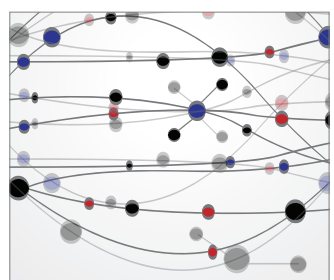

\section{The Scientific} World Journal
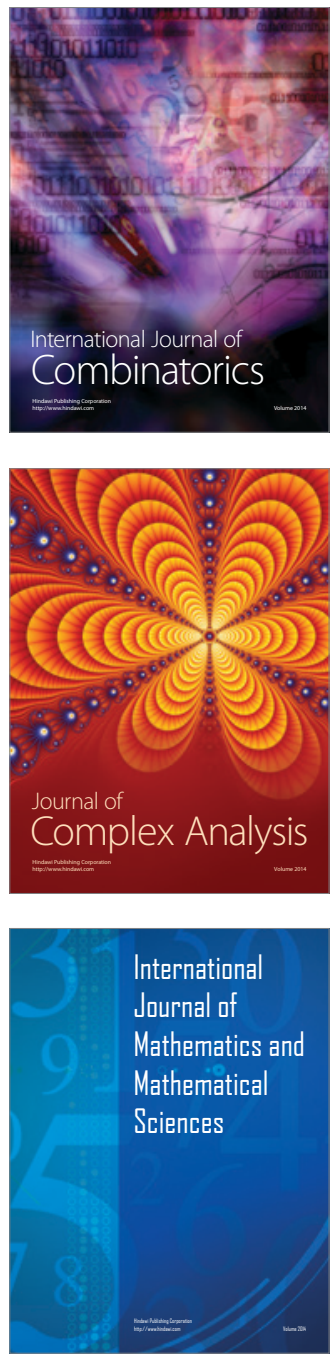
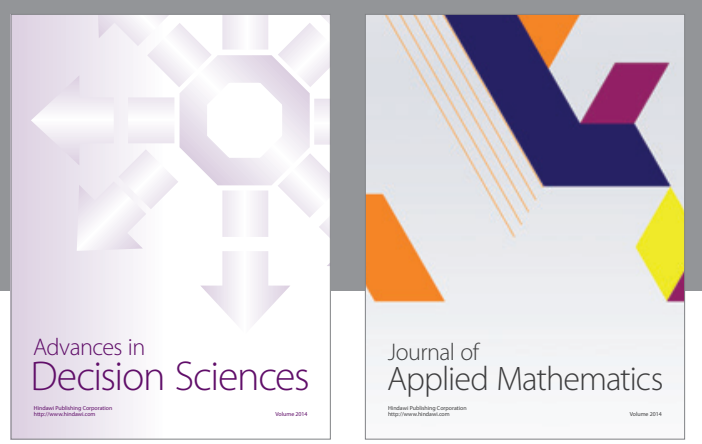

Algebra

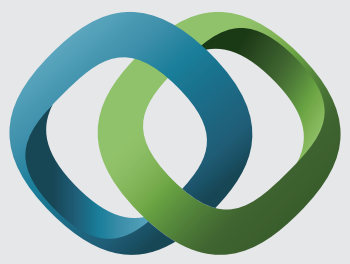

\section{Hindawi}

Submit your manuscripts at

http://www.hindawi.com
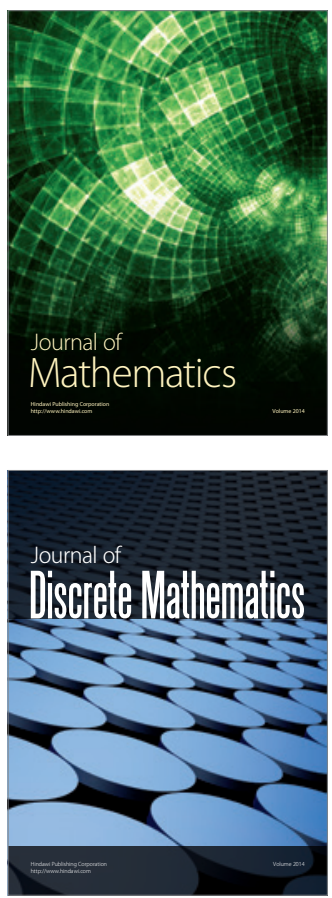

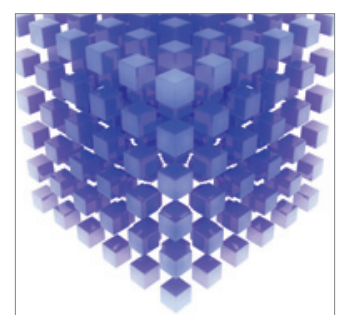

Mathematical Problems in Engineering
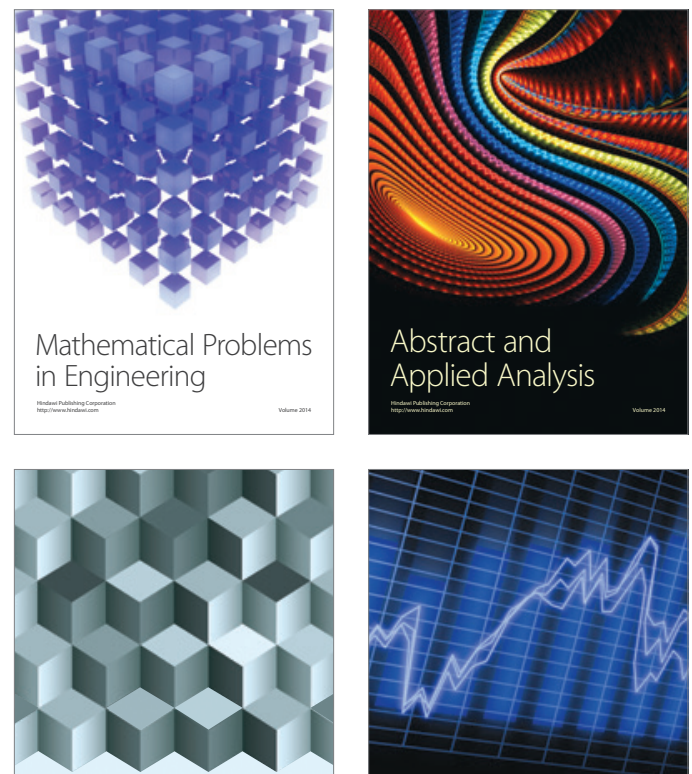

Journal of

Function Spaces

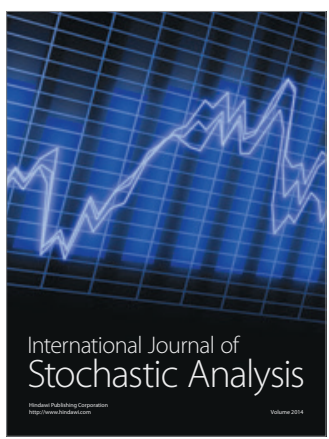

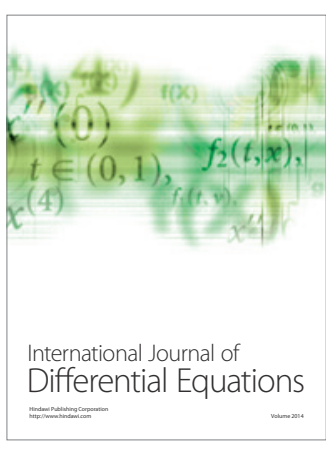
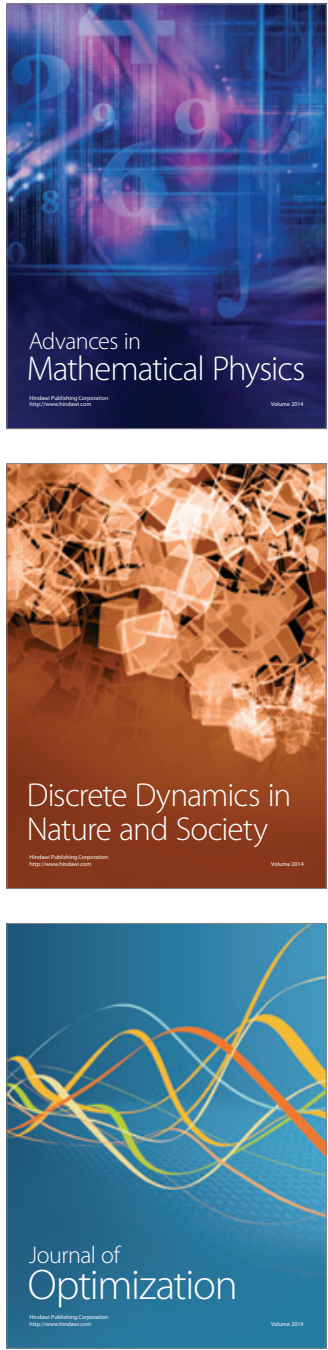